\title{
EXCHANGE SPLITTING OF ZERO-DIMENSIONAL EXCITON LEVELS
}

\author{
S.V. Goupalov and E.L. Ivchenko \\ A.F. Ioffe Physico-Technical Institute \\ Politechnicheskaya 26, 194021 St. Petersburg, Russia
}

\begin{abstract}
A theory of nonanalytic (long-range) exchange interaction between an electron and a hole is developed for zero-dimensional excitons in semiconductor nanostructures. Two particular cases are considered in detail: (i) that of exciton confined in a spherical nanocrystal and (ii) a quantum-well exciton localized as a whole on an anisotropic island of well-width monolayer fluctuation.
\end{abstract}

PACS numbers: $71.70 . \mathrm{Gm}, 73.20 . \mathrm{Dx}, 78.66 .-\mathrm{w}$

\section{Introduction}

The zero-dimensional (OD) microstructures which are also known as quantum dot (QD) structures can be realized in practice in semiconductor nanocrystals embedded in glass matrices and in quantum wells with monolayer-high islands of well-width fluctuations. Optical properties of $0 \mathrm{D}$ systems have been widely studied in the last few years (see $[1,2]$ and references therein). The three-dimensional confinement in quantum dots leads to a discrete exciton-level structure. Taking into account only direct Coulomb interaction between an electron and a hole, the ground-state exciton energy level is degenerate due to the free-carrier spin degeneracy. This degeneracy is, however, removed partially or completely as a result of electron-hole exchange interaction which includes a long- and a short-range contribution. In the present paper we analyze the long-range (or nonanalytic) contribution which is shown to play a crucial role in the interpretation of recent experiments.

\section{Electron-hole exchange Hamiltonian}

In the straightforward derivation [3] the exchange Hamiltonian is presented as a $\operatorname{sum} \sum_{b} \mathcal{H}_{b}$, where $b$ denotes a reciprocal-lattice vector. In the wave-vector-space representation all terms $\mathcal{H}_{b}$ except the one corresponding to $b=0$ are analytic in the limit $\boldsymbol{K} \rightarrow 0$, where $\hbar \boldsymbol{K}$ is the momentum of an electron-hole pair, and only the term $\mathcal{H}_{0}$ behaves nonanalytically at the point $\boldsymbol{K}=0$. Thus, 
the exchange Hamiltonian is usually separated into the analytic and nonanalytic parts, $\mathcal{H}^{\mathrm{A}}=\sum_{b \neq 0} \mathcal{H}_{b}$ and $\mathcal{H}^{\mathrm{NA}}=\mathcal{H}_{0}$, respectively. The latter can be written as

$$
\mathcal{H}_{m^{\prime} n^{\prime}, m n}^{\mathrm{NA}}\left(k_{\mathrm{e}}^{\prime}, k_{\mathrm{h}}^{\prime}, k_{\mathrm{e}}, k_{\mathrm{h}}\right)=\frac{4 \pi \hbar^{2} e^{2}}{\varepsilon_{b} m_{0}^{2} E_{\mathrm{g}}^{2}} \frac{\left(\boldsymbol{K} \boldsymbol{p}_{m^{\prime} \bar{n}^{\prime}}\right)\left(\boldsymbol{K} \boldsymbol{p}_{m \bar{n}}\right)^{*}}{K^{2}} \delta_{K, K^{\prime}},
$$

where $\boldsymbol{K}=k_{\mathrm{e}}+k_{\mathrm{h}}, \boldsymbol{K}^{\prime}=k_{\mathrm{e}}^{\prime}+k_{\mathrm{h}}^{\prime}, k_{\mathrm{e}, \mathrm{h}}$ is the electron or hole wave vector, the indices $m, n$ enumerate the degenerate $\Gamma$-states in the conduction and valence bands, $p_{m \bar{n}}$ is the matrix element of the momentum operator calculated between the electron Bloch functions $|m . k=0\rangle$ and $|\bar{n}, k=0\rangle$ (the hole state $n, k$ and the electron state $\bar{n},-k$ are related by the time inversion operation), $m_{0}$ is the free electron mass, $E_{\mathrm{g}}$ is the band gap, $e$ is the electron charge, $\varepsilon_{b}$ is the high-frequency dielectric constant. Since the selection rules for electron-hole-pair optical excitation are governed by matrix elements of the momentum operator, the nonanalytic part of the electron-hole exchange interaction is non-zero only for the states optically allowed in the dipole approximation. For the $\Gamma_{6} \times \Gamma_{7}$ or $\Gamma_{6} \times \Gamma_{8}$ excitons in the $T_{d}$-symmetry semiconductors, the dipole-active states are characterized by the angular momentum $S=1$ and, in the basis of these states, the nonanalytic Hamiltonian is presented in the following invariant form:

$$
\mathcal{H}^{\mathrm{NA}}\left(k_{\mathrm{e}}^{\prime}, k_{\mathrm{h}}^{\prime}, k_{\mathrm{e}}, k_{\mathrm{h}}\right)=\frac{4 \pi \hbar^{2} e^{2} P_{c v}^{2}}{\varepsilon_{b} m_{0}^{2} E_{\mathrm{g}}^{2}}\left[1-\frac{(\hat{\boldsymbol{K}})^{2}}{K^{2}}\right] \delta_{\boldsymbol{K}, K^{\prime}},
$$

where $P_{c v}^{2}=\sum_{m, n}\left|p_{m \bar{n}}^{\alpha}\right|^{2}, \widehat{I}_{\alpha}$ are the $3 \times 3$ matrices of the projections of the angular momentum $I=1(\alpha=x, y, z)$. The nonanalytic part can be equivalently considered as arising due to the macroscopic electric field induced by the pair excitation. Since this field is a consequence of the exciton polarization, its properties are analogous to that of the depolarization field introduced in the theory of dielectrics.

\section{Excitons in semiconductor nanocrystals}

In semiconductors with $T_{d}$ symmetry the valence band $\Gamma_{8}$ is described by the $4 \times 4$ Luttinger Hamiltonian containing three band parameters $\gamma_{1}, \gamma_{2}$ and $\gamma_{3}$. The ground state of a hole confined in a spherical nanocrystal of the radius $R$ which is smaller than the bulk-exciton Bohr radius, $a_{\mathrm{B}}$, has the symmetry $\Gamma_{8}$ and is characterized by the angular momentum component $n= \pm 3 / 2, \pm 1 / 2$. In the spherical approximation, $\gamma_{2}=\gamma_{3}$, the four ground-state wave functions can be written as $\Psi_{n}(r)=\sum_{n^{\prime}} C_{n^{\prime} n}(r)\left|n^{\prime}\right\rangle$, where $\left|n^{\prime}\right\rangle\left(n^{\prime}= \pm 3 / 2, \pm 1 / 2\right)$ are the Bloch functions,

$$
\widehat{C}(r)=\frac{1}{\sqrt{4 \pi}}\left\{R_{0}(r)-R_{2}(r)\left[\left(J \frac{r}{r}\right)^{2}-\frac{5}{4}\right]\right\},
$$

$J_{\alpha}(\alpha=x, y, z)$ are the angular momentum matrices in the $\Gamma_{8}$ basis, $R_{0}(r)$ and $R_{2}(r)$ are the radial functions introduced in Ref. [4].

In hexagonal crystals one has to add to the Luttinger Hamiltonian the crystal splitting term

$$
\widehat{\mathcal{H}}_{\mathrm{cr}}=-\frac{\Delta_{\mathrm{cr}}}{2}\left(J_{z}^{2}-\frac{5}{4}\right) \text {. }
$$


This term removes the spherical symmetry of the system and leads to a splitting between $n= \pm 3 / 2$ and $n= \pm 1 / 2$ hole states. Thus, the states with definite values of the exciton spin $S$ and its components $0, \pm 1$ are no longer eigenstates of the exciton Hamiltonian. In such case it is convenient to express the nonanalytic exchange contribution (2) in terms of the electron and hole spin matrices. The corresponding Hamiltonian was constructed in Ref. [5]. The analytic exchange interaction has the following form [4]:

$$
\widehat{\mathcal{H}}^{\mathrm{A}}\left(k_{\mathrm{e}}^{\prime}, k_{\mathrm{h}}^{\prime}, k_{\mathrm{e}}, k_{\mathrm{h}}\right)=-\frac{2}{3} \varepsilon_{\mathrm{exch}} \mathrm{a}_{0}^{3}(\sigma J) \delta_{K, K^{\prime}},
$$

where $\sigma_{\alpha}$ are the Pauli matrices acting on the electron spin, $\varepsilon_{\text {exch }}$ is the exchange strength constant, $a_{0}$ is the lattice constant, the factor $a_{0}^{3}$ is introduced for the sake of convenience.

If a value of the crystal splitting $\Delta_{\mathrm{cr}}$ is small as compared to the characteristic confinement energy, the total electron-hole exchange interaction in quantum dots of the radius $R \ll a_{\mathrm{B}}$ is described by the Hamiltonian

$$
\widehat{H}_{\text {exch }}=-\bar{\eta}(\sigma J) \text {. }
$$

Here

$$
\begin{aligned}
& \bar{\eta}=\left(\frac{a_{\mathrm{B}}}{R}\right)^{3}\left[\chi(\beta) \hbar \omega_{\mathrm{TF}}+\frac{\pi}{9} \zeta(\beta) \hbar \omega_{\mathrm{LT}}\right], \\
& \hbar \omega_{\mathrm{TF}}=\frac{2}{\pi}\left(\frac{a_{0}}{a_{\mathrm{B}}}\right)^{3} \varepsilon_{\mathrm{exch}}, \quad \hbar \omega_{\mathrm{LT}}=\frac{4}{\varepsilon_{b} a_{\mathrm{B}}^{3}}\left(\frac{e \hbar P}{m_{0} E_{\mathrm{g}}}\right)^{2},
\end{aligned}
$$

$P=\left|\left\langle S\left|\widehat{p}_{x}\right| X\right\rangle\right|, \chi(\beta)$ and $\zeta(\beta)$ are coefficients dependent on the ratio $\beta=m_{\mathrm{lh}} / m_{\mathrm{hh}}$ of the light- to heavy-hole effective masses. For $\beta=0.3$ we have $\chi(\beta) \approx 0.77$ and $\zeta(\beta) \approx 0.62$. Note that $\hbar \omega_{\mathrm{TF}}$ represents the splitting between the bulk exciton \pm 2 and \pm 1 states induced by the analytic exchange, and $\hbar \omega_{\mathrm{LT}}$ is the longitudinal-transverse splitting for a free exciton propagating perpendicular to the hexagonal axis of the bulk wurtzite-lattice crystal. For CdSe according to Ref. [6] we have $\hbar \omega_{\mathrm{TF}} \approx 0.12 \mathrm{meV}, \hbar \omega_{\mathrm{LT}} \approx 0.95 \mathrm{meV}$ and it follows that the ratio between the second (nonanalytic) and first (analytic) terms amounts about 3.

The splitting, $\Delta_{\mathrm{TF}}^{\mathrm{QD}}(\bar{\eta})$, between the "dark" $( \pm 2)$ and optically-active $( \pm 1)$ states of zero-dimensional exciton is given by

$$
\Delta_{\mathrm{TF}}^{\mathrm{QD}}(\bar{\eta})=2 \bar{\eta}+\frac{\Delta}{2}-\sqrt{4 \bar{\eta}^{2}+\frac{\Delta^{2}}{4}-\bar{\eta} \Delta}
$$

where $\Delta=\Delta_{\mathrm{cr}} v(\beta)$ with $v(\beta) \approx 0.94$ for $\beta=0.3$.

In Refs. $[4,7,8]$ only the first term in Eq. (5) was taken into account. If the nonanalytic exchange contribution is neglected the parameter $\bar{\eta}$ becomes, approximately, four times smaller. Existing experimental data are rather well described by this parameter. Taking the nonanalytic exchange contribution (second term in Eq. (5)) into consideration leads to a considerable discrepancy between theory and experiment. At present there is no satisfactory explanation of this discrepancy and additional theoretical and experimental efforts are needed to resolve this problem. 


\section{Localized excitons in quantum wells}

In this section we consider heavy-hole excitons in a $\mathrm{GaAs} / \mathrm{Al}_{x} \mathrm{Ga}_{1-x} \mathrm{As}(001)$ QW structure. The analytic exchange interaction splits the fourfold degenerate level e1-hh1 $(1 s)$ into a radiative doublet with the spin component $M= \pm 1$ and two close-lying nonradiative singlets. If such an exciton is localized at a rectangular island of the well width fluctuations the radiative doublet is split by the nonanalytic exchange into two sublevels linearly-polarized along the principal axes, $x$ and $y$, of the rectangle. For the island dimensions $L_{x}, L_{y}$ exceeding both the exciton Bohr radius and the $Q W$ width, the splitting between the $|x\rangle$ and $|y\rangle$ states is given by

$$
E_{x}-E_{y}=\frac{\hbar \Gamma_{0}}{S} \sum_{q} \frac{q_{x}^{2}-q_{y}^{2}}{k \sqrt{q^{2}-k^{2}}} f^{2}(q),
$$

where $f(q)$ is the two-dimensional Fourier transform, $\int \mathrm{d} X \mathrm{~d} Y \mathrm{e}^{-\mathrm{i} q \boldsymbol{R}} F(X, Y)$, of the envelope function $F(X, Y)$ describing the in-plane motion of the exciton as a whole, $\boldsymbol{R}=(X, Y)$, the summation in Eq. (7) is performed over $q>k, S$ is the sample area, $k=\left(\omega_{0} / c\right) \sqrt{\varepsilon_{b}}, \omega_{0}$ and $\Gamma_{0}$ are the resonant frequency and the radiative damping rate for a free exciton (with vanishing in-plane wave vector) in a perfect QW [9]. While deriving Eq. (7) both nonanalytic and retarded interactions have been taken into account (the details are given in Ref. [10]).

The function $F(X, Y)$ is found as a solution of the two-dimensional Schrödinger equation

$$
\begin{aligned}
& {\left[-\frac{\hbar^{2}}{2 M}\left(\frac{\partial^{2}}{\partial X^{2}}+\frac{\partial^{2}}{\partial Y^{2}}\right)-V \theta\left(\frac{L_{x}}{2}-|X|\right) \theta\left(\frac{L_{y}}{2}-|Y|\right)\right] F(X, Y)} \\
& =-\varepsilon F(X, Y)
\end{aligned}
$$

where $V$ is the energy difference between the $1 s$-exciton levels in the two perfect QWs differing in width by one monomolecular layer, $\varepsilon$ is the localization energy referred to the free-exciton energy in the thinner $\mathrm{QW}, M$ is the exciton in-plane translational effective mass, $\theta(t)=1$ if $t>0$ and $\theta(t)=0$ if $t<0$. While calculating the splitting given by Eq. (7) we used the approximation of the factorized envelope function and took $F(X, Y)$ as a product $F_{x}(X) F_{y}(Y)$, where $F_{x}(X), F_{y}(Y)$ are found self-consistently from Eq. (8). The localized states $\left|j j^{\prime}\right\rangle$ are labelled by two integer quantum numbers $j j^{\prime}$, e.g., $11,21,22$, etc., describing the exciton in-plane confinement in the $x$ and $y$ directions.

Figure 1 shows the energy and splitting of the ground and excited states as a function of the island dimensions. Note that $E_{j j^{\prime}}=V-\varepsilon_{j j^{\prime}}$ is the localized-exciton energy referred to the free-exciton energy in the wider QW. Recently, Gammon et al. [11] have studied photoluminescence (PL) of GaAs/AlGaAs QWs in the optical near-field regime and measured the PL spectrum of a single quantum dot formed by a large monolayer-high island. They report a fine structure splitting of 20 to $50 \mu \mathrm{eV}$ and linear polarization of the split sublevels for both the ground and excited states of the localized exciton. The sequence of signs of this difference observed for the ground and four excited states of the localized exciton is reproduced in Fig. 1 for values $L_{y}$ lying between 420 and $480 \AA$. Heller and Bockelmann [12] 

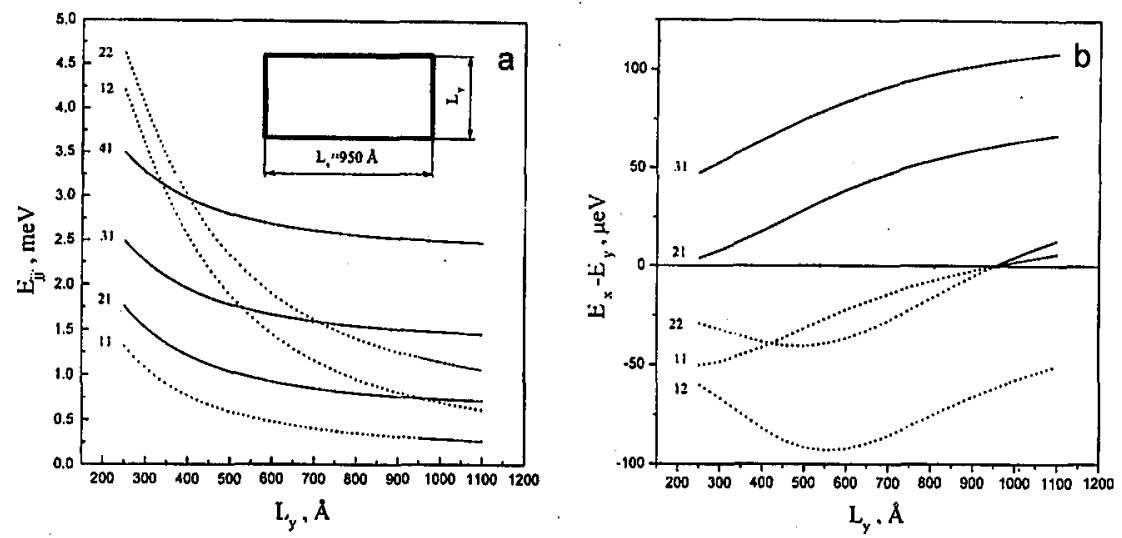

Fig. 1. Energy levels $E_{j j^{\prime}}$ of a localized exciton (a) and splitting $E_{x}-E_{y}$ between localized states $\left|j, j^{\prime}, x\right\rangle$ and $\left|j, j^{\prime}, y\right\rangle$ (b) vs. length $L_{y}$ for a fixed value of $L_{x}=950 \AA$ (see inset) in a GaAs/ $\mathrm{Al}_{0.3} \mathrm{Ga0.7}$ As $28 \AA$ wide quantum well $(N=10)$. The curves show pairs of indexes $j j^{\prime}$ designating the exciton states. The solid and dashed sections of the curves correspond to positive and negative splittings.

observed optical orientation of localized excitons only in the presence of an external magnetic field which can be readily explained assuming a zero-field splitting of the exciton radiative doublet. Baranov et al. [13] studied type-I GaAs/AlAs superlattices using the exciton level-anticrossing spectroscopy. They reported on splitting of the ground localized-exciton state into two sublevels linearly polarized along [110] and [1T0] directions and attributed it to anisotropy of the localizing potential.

\section{Acknowledgments}

We are grateful to O.G. Lublinskaya and I.A. Merkulov for useful discussions. The financial support was provided by the Russian Foundation for Fundamental Research (grant 98-02-18267) and the Volkswagen Foundation.

\section{References}

[1] E.L. Ivchenko, in: Advances in Solid State Physics, Vol. 37, Ed. R. Helbig, Vieweg, Braunschweig/Wiesbaden 1998, p. 125; Phys. Status Solidi A 164, 487 (1997).

[2] P. Lavallard, J. Cryst. Growth 184/185, 352 (1998).

[3] G.L. Bir, G.E. Pikus, Symmetry and Strain-Induced Effects in Semiconductors, Wiley, New York 1974.

[4] Al.L. Efros, M. Rosen, M. Kuno, M. Nirmal, D.J. Norris, M.G. Bawendi, Phys. Rev. B 54, 4843 (1996).

[5] S.V. Goupalov, E.L. Ivchenko, J. Cryst. Growth 184/185, 393 (1998).

[6] V.A. Kiselev, B.S. Razbirin, I.N. Uraltsev, Phys. Status Solidi B 72, 161 (1975).

[7] M. Chamarro, C. Gourdon, P. Lavallard, O. Lublinskaya, A.I. Ekimov, Phys. Rev. $B$ 53, 1336 (1996).

[8] U. Woggon, F. Gindele, O. Wind, C. Klingshirn, Phys. Rev. B 54, 1506 (1996). 
[9] E.L. Ivchenko, Sov. Phys. Solid State 33, 1344 (1991).

[10] S.V. Goupalov, E.L. Ivchenko, A.V. Kavokin, JETP 86, 388 (1998).

[11] D. Gammon, E.S. Snow, B.V. Shanabrook, D.S. Katzer, D. Park, Phys. Rev. Lett. 76, 3005 (1996).

[12] W. Heller, U. Bockelmann, Phys. Rev. B 55, 4871 (1997).

[13] P.G. Baranov, N.G. Romanov, A. Hofstaetter, B.K. Meyer, A. Scharmann, C. Schnorr, W. von Foerster, F.J. Ahlers, K. Pierz, in: Proc. Int. Symp. Nanostructures: Physics and Technology, Eds. Zh. Alferov, L. Esaki, A.F. Ioffe Physico-Technical Institute, St. Petersburg 1997, p. 47. 